\title{
Full Bandwidth Measurement of Supercontinuum Spectral Phase Coherence in Long Pulse Regime
}

\author{
DI YANG, ${ }^{1,2}$ DUC MINH NGUYEN, ${ }^{3,4}{ }^{2}$ ESARE SOCI, ${ }^{4}$ XUAN \\ QUYEN DINH, ${ }^{3,5}$ MING TANG, ${ }^{6}$ and PING PERRY SHUM ${ }^{1,3}$ \\ ${ }^{1}$ OPTIMUS, Photonics Centre of Excellence, Nanyang Technological University, \\ Singapore \\ ${ }^{2}$ Huazhong University of Science and Technology, Wuhan, China \\ ${ }^{3}$ CINTRA CNRS/NTU/THALES, UMI 3288, Singapore \\ ${ }^{4}$ Centre for Disruptive Photonic Technologies, Nanyang Technological \\ University, Singapore \\ ${ }^{5}$ Thales Solutions Asia Pte. Ltd., Singapore \\ ${ }^{6}$ Wuhan National lab for Optoelectronics (WNLO), Huazhong University of \\ Science and Technology, Wuhan, China
}

\begin{abstract}
This article reports that spectral phase coherence in the supercontinuum in long pulse regime can be measured simply and effectively by using an interference technique with the help of a Mach-Zehnder interferometer. It is also demonstrated that chromatic dispersion on the fringe visibility of interference spectral patterns is overcome in the setup. The technique is applied to characterize supercontinuum spectral phase coherence in a highly non-linear optical fiber with different input conditions: unseeded, coherent seeded, and incoherent seeded picosecond pumps. The results confirm the phase coherence characteristic predicted theoretically in previous studies.
\end{abstract}

Keywords modulation instability, non-linear optics, phase coherence, supercontinuum generation

\section{Introduction}

The stability of supercontinuum (SC) broadening in non-linear optical fibers has recently drawn great interest because of important applications in low-noise and high-power broadband source development $[1,2]$, as well as in the research of rogue wave instabilities $[3,4]$. Quantitatively, the stability of SC generation is described through the normalized degree of spectral phase coherence introduced by Dudley and Coen [5] and the statistical higher-order moments that represent shot-to-shot spectral intensity fluctuations [6]. These noise properties have been fully characterized in theoretical simulation for both long and short pump pulses as well as for anomalous and normal dispersion regimes [5-9].

Received 14 October 2014; accepted 17 December 2014.

Address correspondence to Dr. Duc Minh Nguyen, CINTRA CNRS/NTU/THALES, UMI 3288, Research Techno Plaza, 50 Nanyang Drive, Border X Block, Level 6, 637553, Singapore. E-mail: dmnguyen@ntu.edu.sg

Color versions of one or more of the figures in the article can be found online at www. tandfonline.com/ufio. 
In experimentation, although the spectral intensity fluctuation can be measured efficiently in real time by using the time stretch dispersive Fourier transform technique [9], phase information is lost in this measurement. Several interference-based techniques have been proposed to measure the phase noise characteristic in the femto-second regime, such as Young's interference of independently generated SC from two different non-linear fibers $[10,11]$ or delayed pulses Michelson interference method [12]. Experimental results in these works confirm a degradation of the phase coherence when increasing the pulse width in the femtosecond regime, theoretically predicted in [8].

While the dynamics of SC generation in the femto-second regime is originated from soliton fission in the case of anomalous dispersion, the dynamic in the long pulse regime is initiated by noise-driven modulation instability. This leads to a destruction of phase coherence outside the self-phase modulation (SPM) dominated region regardless of the input pulse width. The phase noise characteristic has been previously shown to be improved by adding a weak co-propagating seed to create an initial narrow-band modulation on the pump $[13,14]$. Moreover, the noise properties have been studied in numerical simulation under various modulated conditions of the pump pulses, including coherent continuous-wave (CW) seeded pump and incoherent CW seeded pumps [14, 15]. Another approach to complete characterization of SC coherence using second-order coherence theory of non-stationary light was proposed by Genty et al. [16]. The quasi-coherent and quasi-stationary parts in the SC as well as their relative contributions are theoretically explained in this approach. Although several measurements for phase coherence have been done in the femto-second regime [12], few experimental measurements have been reported in previous studies to confirm the theoretical predictions of phase coherence in the long pulse regime.

This article makes a detailed review of the delayed-pulses interference-based technique and shows that this technique is efficient and reliable to measure SC phase fluctuations in the long pulse modulation instability regime. Also demonstrated, both in simulation and experimentally, is a direct relation between the free fringe visibility of the averaged interference spectra of two delayed subsequent pulses and the degree of spectral phase coherence. A particular originality of this work is that the modulation instability spectral phase coherence is measured experimentally at different pulse widths of picosecondpulsed pumps by the new technique. The measurement is implemented on the beginning of the picosecond regime where the phase coherence has not been totally destroyed. It is also demonstrated that the influence of chromatic dispersion (CD) on the measured results is negligible. Finally, experimental perspectives of the technique are shown by calculating fringe visibility in numerical simulation that models the proposed technique under various modulated conditions of the input pulses: coherent and incoherent seeded pulsed pumps. The results show good agreement between simulation and experiment and confirm the phase coherence characteristics predicted theoretically in previous studies.

\section{Experimental and Numerical Results}

\subsection{Experimental Setup}

The experimental setup is shown in Figure 1. Picosecond pulses at 1,564 nm and a 500$\mathrm{MHz}$ repetition rate are amplified by an erbium-doped fiber amplifier (EDFA; ACCELINK, TV-23) and injected into a 50-m highly non-linear fiber (HNLF; YOFC, NL-1550-POS) through a polarization controller (PC). The HNLF has a non-linear coefficient $\gamma$ of $10.5 \mathrm{~W}^{-1} \mathrm{~km}^{-1}$, second-order dispersion parameter $\beta_{2}$ of $-3.2 \mathrm{ps}^{2} \mathrm{~km}^{-1}$, 


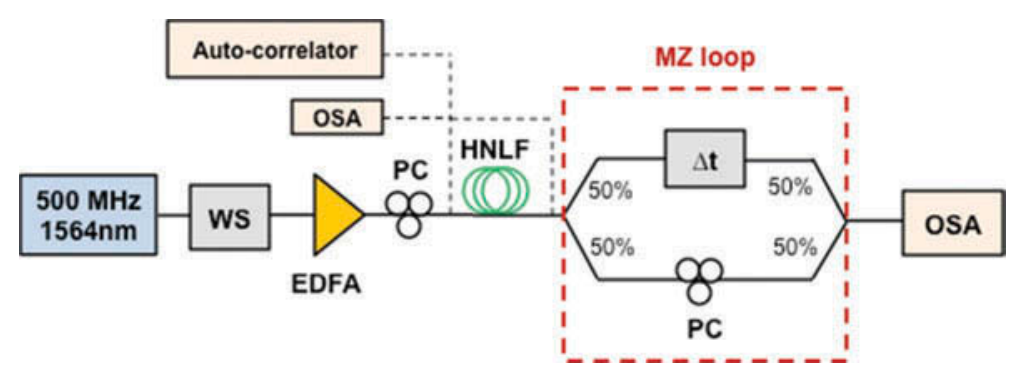

Figure 1. Experimental setup (WS: waveshaper).

and third-order dispersion parameter $\beta_{3}$ of $0.042 \mathrm{ps}^{3} \mathrm{~km}^{-1}$. The corresponding zero dispersion wavelength is at $1,455 \mathrm{~nm}$. The pulses at the input of the HNLF are characterized by an auto-correlator and an optical spectrum analyzer (OSA; ANDO, AQ6317B) to have a time-bandwidth product of 0.45 . SC spectra at the HNLF output are then recorded by the OSA. To measure the spectral phase noise, the generated SC trains are injected into a Mach-Zehnder (MZ) interferometer consisting of two unequal arms. The optical path difference between the two arms is nearly equal to the distance between consecutive pulses. In this particular experiment, the longer arm is set to have an exact delay time of $2 T_{0}+\Delta t$, where $T_{0}(\sim 2 \mathrm{~ns})$ is the period of picoseconds train pulses, and $\Delta t$ is an adjustable delay time that can vary with an interval of $0.01 \mathrm{ps}$ by using an optical delay line (ODL-100-11-1550-9). The interference spectra between the pulse in the short arm and its second consecutive pulse with delay time $\Delta t$ in the longer arm are finally retrieved by the OSA. The spectral phase noise can be analyzed from the interference spectra as explained next.

\subsection{Theory and Numerical Approach}

To identify the relation between the MZ interference spectra with the spectral phase noise, consider $E_{n}(t)$ as the amplitude of the $n$th pulse at the output of the short arm. Since there exists a delay time of $2 T_{0}+\Delta t$ between the two arms, the pulse in the short arm will overlap with the $(n-2)$ th pulse in the long arm. The signal at the end of the MZ interferometer will be

$$
E(t)=E_{n}(t)+E_{n-2}(t+\Delta t) e^{i \omega_{0}\left(2 T_{0}+\Delta t\right)} .
$$

Note that Eq. (1) uses a time coordinate co-moving with the $n$th pulse. The Fourier transform of the temporal amplitude will be then

$$
\tilde{E}(\omega)=\tilde{E}_{n}(\omega)+\tilde{E}_{n-2}(\omega) e^{i\left[\omega \Delta t+\omega_{0}\left(2 T_{0}+\Delta t\right)\right]},
$$

where $\tilde{E}(\omega)$ is the Fourier transform of $E(t)$. To take into account the intensity and phase noise, the $(n-2)$ th pulse is related with the nth pulse by

$$
\tilde{E}_{n-2}=\tilde{E}_{n}(1+\Delta E) e^{i \Delta \phi}
$$

where $\Delta E$ and $\Delta \varphi$ are responsible for the intensity and phase fluctuation, respectively. The final overlapping spectrum $\left(\tilde{I}=\tilde{E} \tilde{E}^{*}\right)$ is then given by 


$$
\begin{aligned}
\tilde{I}= & \tilde{I}_{n}\left(2+2 \Delta E+\Delta E^{2}\right)+2 \tilde{I}_{n} \cos \left[\omega \Delta t+\omega_{0}\left(2 \Delta T_{0}+\Delta t\right)+\Delta \phi\right] \\
& +2 \tilde{I}_{n} \Delta E \cos \left[\omega \Delta t+\omega_{0}\left(2 \Delta T_{0}+\Delta t\right)+\Delta \phi\right]
\end{aligned}
$$

The second and last terms on the right-hand side (r.h.s.) of Eq. (4) lead to interference patterns of the spectrum at the end of the MZ loop. Since the last term is one order smaller than the second term, interference characteristics, such as the period and the maximum peak position, will be mainly governed by phase difference $\Delta \varphi$ and time delay $\Delta t$. Particularly, interference spectra have period of $2 \pi / \Delta t$, and the maximum peaks are situated at frequency of $\left(2 \pi m-\left(\omega_{0}\left(2 \Delta T_{0}+\Delta t\right)+\Delta \varphi\right)\right) / \Delta t$. This means that spectral phase noise $\Delta \varphi$ leads to a jitter of the interference pattern, thus reducing the free fringe visibility of the interference spectrum measured by the OSA, which is an average of thousands of shot-to-shot interference spectra. In consequence, the free fringe visibility of the averaged interference spectrum can be used to quantify the spectral phase noise.

To model the statistics of SC generation, including dispersion, self-steepening, and Raman scattering, generalized stochastic non-linear Schrödinger equation (GNLSE) equations [17] are used:

$$
\frac{\partial A}{\partial z}-i \sum_{k \geq 2} \frac{i^{k} \beta_{k}}{k !} \frac{\partial^{k} A}{\partial t^{k}}=i \gamma\left(1+\frac{i}{\omega_{0}} \frac{\partial}{\partial t}\right) \times\left[A(z, t) \int_{-\infty}^{t} R\left(t^{\prime}\right)\left|A\left(z, t-t^{\prime}\right)\right|^{2} d t^{\prime}\right],
$$

where $A=A(z, t)$ is the electric field envelope, $\beta_{k}$ denotes the $k$-order dispersion coefficients at center frequency $\omega_{0}$, and $\gamma$ is the third-order non-linear coefficient. The response function $R(t)=\left(1-f_{R}\right) \delta(t)+f_{R} h_{R}(t)$ presents both instantaneous electronic and delayed Raman contributions, in which $f_{R}=0.18$ for the silica glass and $h_{R}$ is the experimentally determined Raman response of silica fibers.

\subsection{Results}

An important statistical parameter that is usually used to quantify the phase noise is the normalized degree of spectral phase coherence introduced by Dudley and Coen [5] and defined as

$$
g_{12}^{(1)}(\omega)=\frac{\left\langle\tilde{E}_{i}^{*}(\omega) E_{j}(\omega)\right\rangle_{i \neq j}}{\left\langle|E(\omega)|^{2}\right\rangle}
$$

where the $<>_{i \neq j}$ operator denotes averaging over an ensemble of independent SC spectra $\tilde{E}_{i}(\omega)$. A detailed study of this relation is now made in SC generation with different input conditions. First investigated are the phase noise characteristics of SC spectra generated from picosecond pulses described in the experimental setup in Figure 1. Since SC generation in the picosecond regime is initiated by noise-driven modulation instability, the spectral phase coherence should be strongly degraded or totally destroyed. In the present experiment, cases where the phase coherence has not been totally destroyed yet is considered by adjusting the pulse width at only several picoseconds. Particularly, a tunable filter (Finisar Waveshaper 4000S) is used to set the pulse width to be 4.3 and 2 ps with corresponding powers of 14 and $7 \mathrm{~W}$, respectively. The mechanism of coherence can be estimated through input soliton order $N=\left(\gamma P_{0} T_{0}^{2} /\left|\beta_{2}\right|\right)^{1 / 2}$ and soliton 
fission distance $L_{f i s s} \sim L_{D} / N$, where $L_{D}=T_{0}^{2} /\left|\beta_{2}\right|$ is the dispersion length [8]. When the fiber length is smaller than the fission distance, and pump pulses propagate over a fraction of a soliton period and reach the maximum broadening before soliton fission can occur, modulation instability gain of the noise background will take place, leading to degradation of coherence. In the present experiment, an input 4.3-ps pulse is associated with $N \sim 29$ and $L_{\text {fiss }} \sim 199 \mathrm{~m}$, whereas for a 2-ps pulse, $N \sim 10$ and $L_{\text {fiss }} \sim 125 \mathrm{~m}$. Since these calculated fission distances are longer than the fiber length of $50 \mathrm{~m}$ in the experiment, and the characteristic scale of the SPM $\left(\sim 14 \mathrm{~m}\right.$ calculated using formula $\left.L_{N L}=1 / \gamma P\right)$, the SC generation will be in the modulation instability regime. Moreover, the coherence degree in the case of 2-ps pulses should be improved comparing to the other due to the smaller fission distance.

Figure 2a shows experimental and numerical results for SC spectra at the end of the HNLF, interference spectra after the MZ loop, free fringe visibility, and simulated spectral phase coherence for the case of input pulse width of 4.3 ps. In Figure 2a (a4), 500 individual realizations of SC spectra at the end of the HNLF with different initial random

(a) $\mathrm{FWHM}=4.3 \mathrm{ps}$

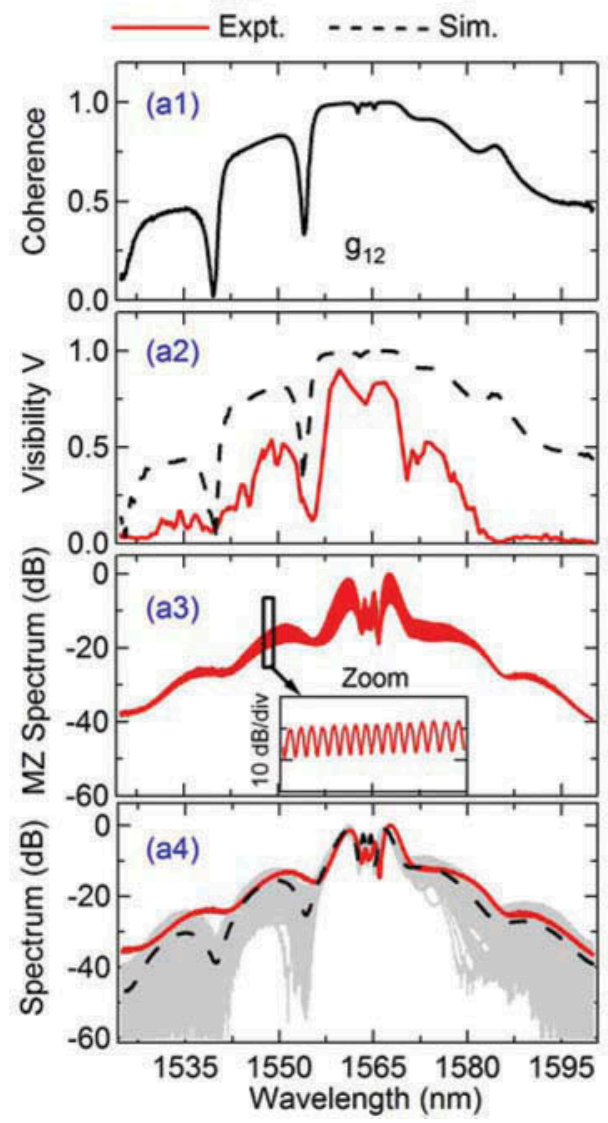

(b) $\mathrm{FWHM}=2 \mathrm{ps}$

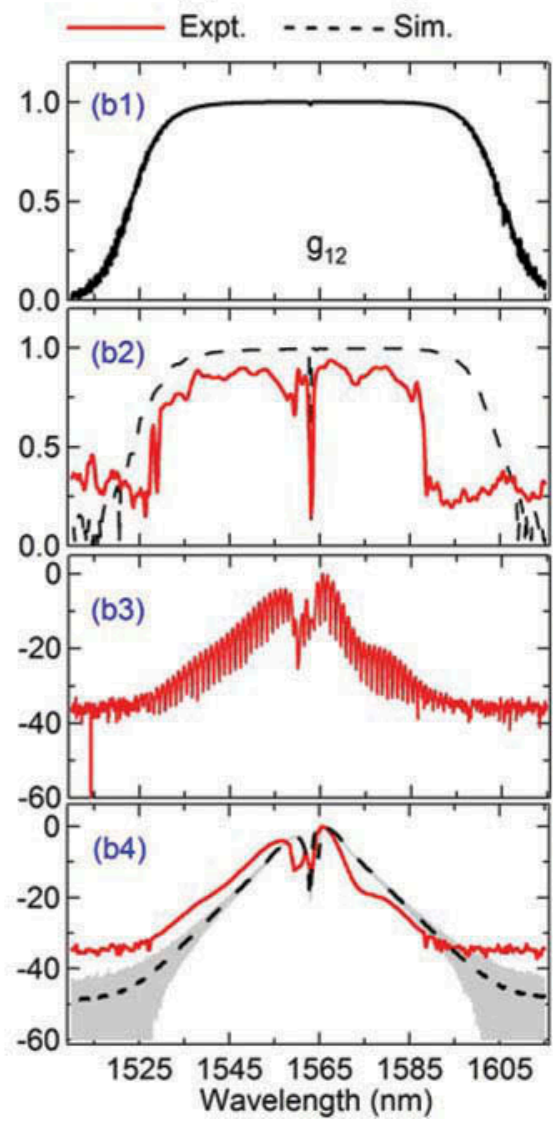

Figure 2. Experimental and numerical results of SC characteristics for two cases: (a) input pulse width of 4.3 ps and (b) input pulse width of 2 ps. From bottom to top: SC spectra, MZ interference spectra, free fringe visibility $V$, and coherence. 
noise from the simulation ensemble as well as their mean are plotted. Note that in simulation, input EDFA noise, asymmetric properties of input pulses, and single-mode fiber (SMF) segment $\left(D=17 \mathrm{ps} . \mathrm{nm}^{-1} \mathrm{~km}^{-1}, \gamma=1.3 \mathrm{~W}^{-1} \mathrm{~km}^{-1}\right)$ before the HNLF are taken into account. MZ spectra in Figure 2a (a3) has interference patterns with a period of 0.124 $\mathrm{nm}$ that corresponds to the delay time of $405 \mathrm{ps}$, as explained in Eq. (4). Good agreements between the simulation and experiment in Figure 2 demonstrate the ability to use the proposed numerical model to study statistical properties, such as intensity fluctuation and spectral phase coherence. Degradation of the measured fringe visibility compared to that simulated in Figure 2a (a2) is due to misalignment of the polarization state between two $\mathrm{MZ}$ arms and pump pulses jitter. Another important reason causing this difference between the experiment and simulation is the limitation of the sensitivity of the OSA due to the fact that powers of wavelength components decrease rapidly when they are far from the center wavelength, as can be seen in Figure 2a (a4). Particularly, the intensity at $1,585 \mathrm{~nm}$ is 30 $\mathrm{dB}$ smaller than the center wavelength intensity. Note that spectra in Figure 2 are normalized to the maximum intensity.

Despite this small degradation, direct correspondence between the visibility and spectral phase coherence are demonstrated through their perfect similarity as observed in Figure 2a (a2 and a1) in which phase coherence is good in the SPM region (1,555-1,570 $\mathrm{nm})$ but degrades quickly outside. Influence of noise-driven modulation instability and soliton fission in SC generation to the phase coherence is experimentally demonstrated in Figure $2 \mathrm{~b}$, where the input pulse width is reduced to $2 \mathrm{ps}$. In this case, SC generation is closer to the femtosecond regime and soliton fission plays a more important role. In consequence, good phase coherence is maintained over a broad range from 1,530 to 1,600 $\mathrm{nm}$, which is much broader than the previous more direct correspondence between the fringe visibility and spectral phase coherence.

A positive point in the experimental setup is that influences of the overlapping between different spectral frequencies caused by $C D$ on the fringe visibility are negligible. This is due to the structure of the MZ loop used in the present setup that consists of a segment of SMF of length $L_{1}$ in the first arm and a segment of SMF of length $L_{2}$ associated with a free space delay line of length $d$ in the second arm. The condition $L_{2}>L_{1}$ assures that internal interference between spectral frequencies caused by CD can be ignored. To demonstrate this, consider interference of a continuous broadband signal after propagating through the $\mathrm{MZ}$ loop. The spectrum of the combined beam of electric fields $E_{1}$ and $E_{2}$ in two arms can be calculated as done in [18]

$$
I(\omega)=\left|E_{1}+E_{2}\right|^{2} \tilde{1}+\cos (\phi)
$$

where

$$
\begin{gathered}
\phi(\omega)=\left[\beta_{1}\left(\omega_{0}\right) \Delta L+T\right]\left(\omega-\omega_{0}\right)+\frac{1}{2} \beta_{2}\left(\omega_{0}\right) \Delta L\left(\omega-\omega_{0}\right)^{2}+\ldots, \\
\Delta L=L_{2}-L_{1}, \quad \beta_{1}=\frac{\partial \beta}{\partial \omega}, \quad \beta_{2}=\frac{\partial \beta_{1}}{\partial \omega}, \quad T=\frac{d}{c}
\end{gathered}
$$

From Eq. (6), the spectrum $I(\omega)$ will show local maximum and minimum when $\phi=n \pi$. The crucial condition that governs if the interference caused by CD is important or can be ignored is that the term $A=\beta_{1}\left(\omega_{0}\right) \Delta L+T$ on the r.h.s. of Eq. (7) can reach 0 by adjusting the optical delay $T$ or not. In this particular setup, $A$ is always much 
different from 0 because $\Delta L>0$; the frequency period of the fringe will be $\Delta \omega=$ $\pi /\left(\beta_{1}\left(\omega_{0}\right) \Delta L+T\right) \sim \pi \mathrm{v}_{g} / \Delta L$, where $v_{g}$ is the group velocity. This period is calculated to be smaller than $0.0008 \mathrm{~nm}$ in this case, which is much smaller than the OSA resolution of $0.015 \mathrm{~nm}$ and the fringe period caused by interference between two delayed pulses observed in this experiment $(>0.1 \mathrm{~nm})$. The influence of CD in the MZ loop on the fringe visibility is therefore negligible.

In the explanation of Eq. (4), it is seen that spectral intensity fluctuations also influence the interference patterns and, thus, the measured visibility. To illustrate more clearly that the contribution of the intensity fluctuation to the visibility is much smaller than the one from phase coherence, Figure 3 shows a comparison between the visibility and intensity and phase noise characteristics for three cases: unseeded (Figure 3a), coherent CW seeded (Figure 3b), and incoherent seeded (Figure 3c) SC generation. Although spectral broadening and fluctuation of these three cases are well investigated in other publications $[6,7,16]$, a confirmation of phase noise characteristic by measuring fringe visibility of interference spectra has not been done. Subfigures from the bottom to the top of Figure 3 represent, respectively, spectral intensity, coefficient of variance $C v$, spectral phase coherence $g_{12}$, and fringe visibility $V$. Definition of coefficient of variance $C v$ is presented in [6], and the corresponding physical significance is related to intensity fluctuations. The input pulse width is increased to $6 \mathrm{ps}$ to assure that the SC mechanism is completely in the long pulse regime, where SC generation is initiated mainly by the noise-driven modulation instability (MI). The input pulse and seed powers are, respectively, $7 \mathrm{~W}$ and $0.7 \mathrm{~mW}$. The incoherent seed is an arbitrary spontaneous emission (ASE) signal with a bandwidth of $1 \mathrm{~nm}$. As

(a) No seed

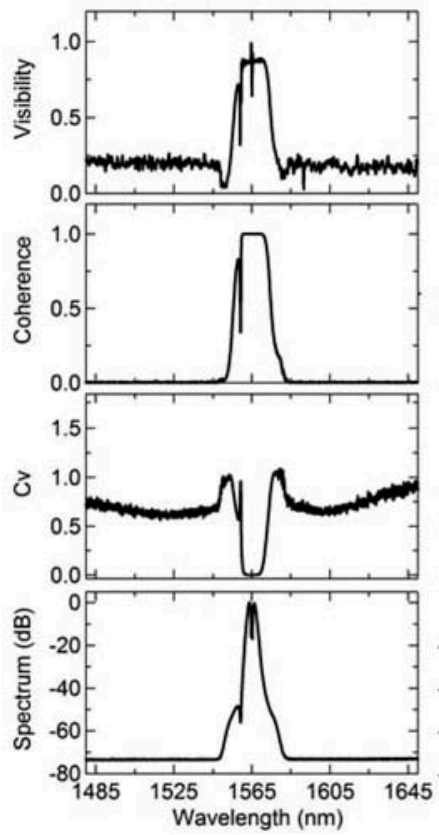

(b) $\mathrm{CW}$ seed at $1554 \mathrm{~nm}$

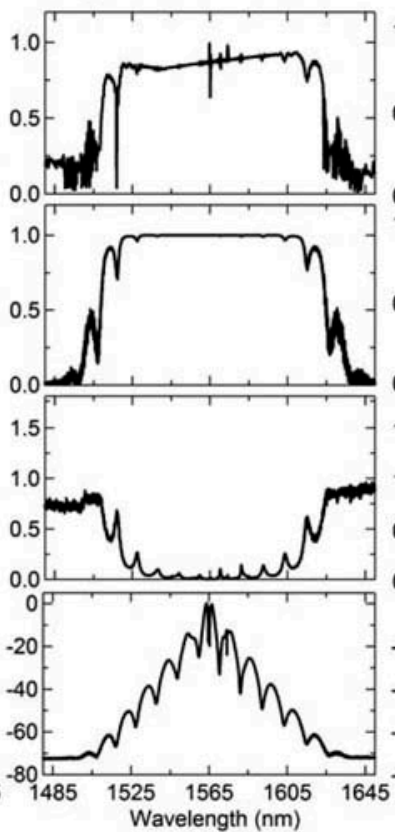

(c) ASE seed at $1554 \mathrm{~nm}$

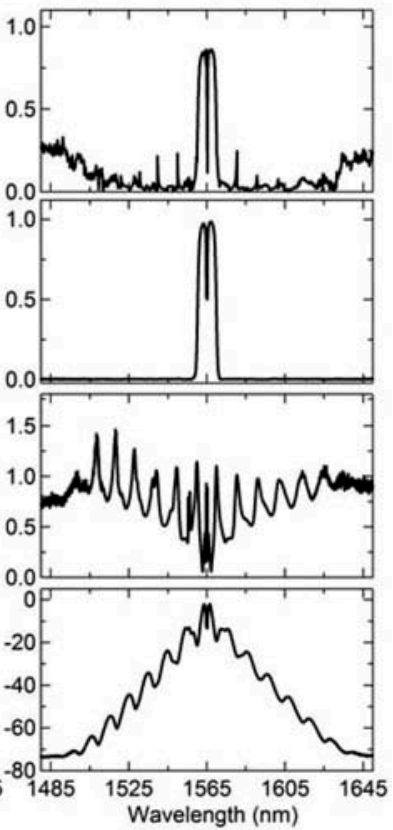

Figure 3. Subfigures from bottom to top represent, respectively, spectral intensity, coefficient of variance $C v$, spectral phase coherence $g_{12}$, and fringe visibility $V$ for three cases: no seeded, coherent $\mathrm{CW}$ seeded, and ASE incoherent seeded SC generation. The input pulse width is $6 \mathrm{ps}$. 
described in $[7,8]$, the unseeded SC spectrum in this regime has high intensity fluctuations (high $C v$ ) and extremely low phase coherence (low $g_{12}$ ) outside the SPM region (see Figure 3a). Inversely, when the pump is seeded by a coherent $\mathrm{CW}$, both intensity and spectral noise are much improved, as shown in Figure $3 \mathrm{~b}$ (low $C v$ and high $g_{12}$ ). In these figures, very good agreement is also seen between visibility $V$ calculated from MZ interference spectra and the phase coherence. The ability to use the fringe visibility for representing the phase coherence regardless of intensity fluctuation is clearly demonstrated in Figure 3c, where the pump is seeded by an incoherent ASE seed. In this case, although the intensity fluctuation expressed through $C v$ is much more improved compared to the unseeded case in Figure 3a, fringe visibility $V$ still agrees well with the phase coherence, which indicates a total destruction of the phase coherence outside the SPM region. Calculated values of the visibility in Figure 3 confirm theoretical estimation of phase noise properties of seeding SC generation in previous publications $[6,7,15]$.

\section{Conclusions}

It has been shown that the delayed-pulses-based interference technique is efficient and reliable to determine phase noise properties of SC. By using an MZ interferometer with length difference between two arms equal to several pulse periods, the average of thousands of interference spectra between two different particular pulses can be recorded. Numerical and experimental results not only demonstrate great correspondence between the fringe visibility of averaged interference spectra and spectral phase coherence but also show that influence of intensity fluctuations to the measured visibility can be ignored. Finally, the visibility for different SC generation cases was calculated-unseeded, coherent $\mathrm{CW}$, and incoherent ASE seeded SC generation - and the results confirm the phase properties numerically calculated in previous publications.

\section{References}

1. Boucon, A., Fotiadi, A. A., Mégret, P., Maillotte, H., and Sylvestre, T. 2008. Low-threshold allfiber $1000 \mathrm{~nm}$ supercontinuum source based on highly non-linear fiber. Optics Communications 281:4095.

2. Genty, G., and Dudley, J. M. 2009. Route to coherent supercontinuum generation in the long pulse regime. IEEE Journal of Quantum Electronics 45:1331-1335.

3. Solli, D. R., Ropers, C., Koonath, P., and Jalali, B. 2007. Optical rogue waves. Nature 450:1054-1057.

4. Akhmediev, N., Dudley, J. M., Solli, D. R., and Turitsyn, S. K. 2013. Recent progress in investigating optical rogue waves. Journal of Optics 15:060201.

5. Dudley, J. M., and Coen, S. 2002. Coherence properties of supercontinuum spectra generated in photonic crystal and tapered optical fibers. Optics Letters 27:1180-1182.

6. Sorensen, S. T., Bang, O., Wetzel, B., and Dudley, J. M. 2012. Describing supercontinuum noise and rogue wave statistics using higher-order moments. Optics Communications 285:2451.

7. Li, Q., Li, F., Wong, K. K.Y., Lau, A. P. T., Tsia, K. K., and Wai, P. K. A. 2011. Investigating the influence of a weak continuous-wave-trigger on picosecond supercontinuum generation. Optics Express 19:13757-13769.

8. Dudley, J. M., Genty, G., and Coen, S. 2006. Supercontinuum generation in photonic crystal fiber. Reviews of Modern Physics 78:1135.

9. Wetzel, B., Stefani, A., Larger, L., Laourt, P. A., Merolla, J. M., Sylvestre, T., Kudlinski, A., Mussot, A., Genty, G., Dias, F., and Dudley, J. M. 2012. Real-time full bandwidth measurement of spectral noise in supercontinuum generation. Scientific Reports 2:882. 
10. Bellini, M., and Hänsch, T. W. 2000. Phase-locked white-light continuum pulses: Toward a universal optical frequency comb synthesizer. Optics Letters 25:1049-1051.

11. Gu, X., Kimmel, M., Shreenath, A. P., Trebino, R., Dudley, J. M., Coen, S., and Windeler, R. S. 2003. Experimental studies of the coherence of microstructure-fiber supercontinuum. Optics Express 11:2697-2703.

12. Türke, D., Pricking, S., Husakou, A., Teipel, J., Herrmann, J., and Giessen, H. 2007. Coherence of subsequent supercontinuum pulses generated in tapered fibers in the femtosecond regime. Optics Express 15:2732-2741.

13. Cheung, K. K. Y., Zhang, C., Zhou, Y., Wong, K. K. Y., and Tsia, K. K. 2011. Manipulating supercontinuum generation by minute continuous wave. Optics Letters 36:160-162.

14. Sørensen, S. T., Larsen, C., Møller, U., Moselund, P. M., Thomsen, C. L., and Bang, O. 2012. Influence of pump power and modulation instability gain spectrum on seeded supercontinuum and rogue wave generation. Journal of the Optical Society of America B 29:2875-2885.

15. Nguyen, D. M., Godin, T., Toenger, S., Combes, Y., Wetzel, B., Sylvestre, T., Merolla, J.-M., Larger, L., Genty, G., Dias, F., and Dudley, J. M. 2011. Incoherent resonant seeding of modulation instability in optical fiber. Optics Letters 38:5338-5341.

16. Genty, G., Surakka, M., Turunen, J., and Friberg, A. T. 2011. Complete characterization of supercontinuum coherence. Journal of the Optical Society of America B 28:2301-2309.

17. Agrawal, G. P. 2012. Chapter 2.3. In: Nonlinear Fiber Optics, 5th ed. Oxford, UK: Academic Press.

18. Shang, H.-T. 1981. Chromatic dispersion measurement by white-light interferometry on metrelength single mode optical fibers. Electronics Letters 17:603-605.

\section{Biographies}

Di Yang was born in Hu Bei Province, China, in 1987. She is currently pursuing her Ph.D. in Huazhong University of Science and Technology, Wuhan, China. Her main research focuses on investigation of non-linear optics in fiber lasers.

Duc Minh Nguyen received his Ph.D in non-linear optics in 2011 at University of Rennes 1 in France. From November 2011 to October 2013, he worked at the Institute of Femto-st, University of Franche-Comté, France, as a post-doctoral research fellow; his research during this period focused on the study of the amplification parametric effect and the Brillouin effect in the photonic crystal fibers as well as their applications, such as distributed Brillouin sensors. He has also worked on intriguing links between ocean rogue waves and non-linear optics, more specifically, studying different kinds of soliton (Perregine, Kuznetsov-Ma, etc.) in non-linear Schrödinger equations as well as real-time measurements techniques of ultrashort laser pulses. He is currently a senior research fellow in the CNRS International Thales Research Alliance (CINRA) and a research project leader at Centre for Disruptive Photonic Technologies (CDPT), Nanyang Technological University, Singapore.

Cesare Soci received both his Laureat degree (2000) and Ph.D. in physics (2005) from the University of Pavia, Italy. From 2005 to 2006, he was a postdoctoral researcher at the Center for Polymers and Organic Solids at University of California, Santa Barbara, and from 2006 to 2009 at the Electrical and Computer Engineering Department at University of California, San Diego. In 2009, he joined Nanyang Technological University (NTU) as an assistant professor at the School of Physical and Mathematical Sciences (SPMS) and Electrical and Electronic Engineering (EEE). He is currently the deputy director of the Centre for Disruptive Photonic Technologies. 
Xuan Quyen Dinh received his Ph.D in physics in 2007 at Ecole normale Supérieur de Cachan in France. He is now the deputy director of CNRS International Thales Research Alliance (CINRA) at Nanyang Technological University, Singapore.

Ming Tang received his B. Eng. from Huazhong University of Science and Technology (HUST), Wuhan, China, in 2001 and his Ph.D. from Nanyang Technological University (NTU), Singapore, Singapore, in 2005. His postdoctoral research in Network Technology Research Centre (NTRC) was focused on the optical fiber amplifiers, high-power fiber lasers, non-linear fiber optics, and all-optical signal processing. From February 2009, he was with the Tera-Photonics Group led by Prof. H. Ito in Riken, Wako, Japan, as a research scientist conducting research on terahertz-wave generation, detection, and application using non-linear optical technologies. Since March 2011, he has been a professor in the School of Optoelectronics Science and Engineering, Wuhan National Laboratory for Optoelectronics, HUST, Wuhan, China. He has published more than 70 technical papers in international recognized journals and conferences, and he serves as a regular reviewer for such journals as IEEE Journal of Quantum Electronics, IEEE Journal of Lightwave Technology, IEEE Photonics Technology Letters, and Optical Society of America (OSA) publications. He has been a member of the Lasers and Electro-Optics Society (LEOS; now IEEE Photonics Society) since 2001.

Ping Perry Shum received his B. Eng. and Ph.D. in electronic and electrical engineering from University of Birmingham, Birmingham, United Kingdom, in 1991 and 1995, respectively. He was an honorary postdoctoral research fellow in University of Birmingham. In 1996, he was involved in research of semiconductor laser and highspeed optical laser communication in the Department of Electrical and Electronic Engineering, Hong Kong University, as a visiting research fellow. In July 1997, he joined the Department of Electronic Engineering, Optoelectronics Research Centre, City University of Hong Kong, Hong Kong. In 1999, he joined the School of Electrical and Electronic Engineering, Nanyang Technological University, Singapore. Since 2002, he has been appointed as the director of the Network Technology Research Centre, Nanyang Technological University. From 2010, he was an adjunct professor in Wuhan National Laboratory for Optoelectronics (WNLO) and School of Optoelectronics Science and Engineering, Huazhong University of Science and Technology, China. He has published more than 400 international journal and conference papers. His research interests include optical communications, non-linear waveguide modeling, and fiber-based technology. He received the Singapore National Academy of Science Young Scientist Award in 2002 for his contributions on next-generation optical communication technology. 